\title{
Indirect Search for Dark Matter with the ANTARES Neutrino Telescope
}

\author{
Pascal GAY*广 \\ $L P C$ \\ E-mail: pascal.gayein2p3.fr
}

The ANTARES Collaboration is now operating the largest water Cherenkov neutrino telescope in the Nothern hemisphere. The apparatus, completed in May 2008, comprises 12 detection lines and a multidisciplinary instrumentation line installed at a depth of about $2500 \mathrm{~m}$ in the Mediterranean Sea offshore from France.

The goals of ANTARES are among others the search for astrophysical neutrino point sources and for neutrinos produced in self-annihilation of dark matter particles. Likely sources of the latter type of neutrino emission would be the Sun and the Galactic Centre, where dark matter particles from the galactic halo are expected to accumulate.

Prior to its completion, ANTARES has been taking data for more than a year in an intermediate setup with a five and a ten line detector configuration. Results on the search for dark matter annihilation in the Sun with the data recorded in 2007 are presented, as well as sensitivity studies on Dark Matter searches with the full ANTARES detector

Identification of Dark Matter 2010

July 26 - 302010

University of Montpellier 2, Montpellier, France

*Speaker.

${ }^{\dagger}$ on behalf of the ANTARES collaboration 


\section{Introduction}

There is solid evidence showing that majority of the matter content of the Universe is of nonbaryonic specie and is in favor of the existence of cold dark matter. Weakly Interacting Massive Particles (WIMPs) are considered the main candidate for cold dark matter. One of the most favorite extension of the Standard Model are the models based on the Super-Symmetry. And among the new particle involved, the neutralinos which are the lightest supersymmetric stable particle in most models due to the conservation of R- parity, are Dark Matter candidates. In the supersymmetric dark matter scenario, neutralinos have been copiously produced in the beginning of the universe. A relic neutralino density would be present in the universe today due to the expansion of the Universe. Then, these relic neutralinos could accumulate in massive celestial bodies in the Universe. WIMPs are expected to scatter elastically in massive objects like the Sun or the Earth and become gravitationally trapped. They would annihilate producing standard model particles, among which neutrinos. Such neutrinos would be observed neutrino telescopes like ANTARES as an indirect probe of the Dark Matter.

\section{The ANTARES neutrino telescope}

ANTARES (Astronomy with a Neutrino Telescope and Abyss environmental RESearch) [1] is a submarine Cerenkov detector located in the Mediterranean sea, $40 \mathrm{~km}$ off Toulon (France), at a depth of 2475 metres. It is a Cherenkov neutrino telescope comprising 12 detection lines in an approximately cylindrical layout and a surface of $0,06 \mathrm{~km}^{2}$. Each line carries 75 photomultipliers tubes (PMTs) located inside pressure resistant spheres. Each storey contains 3 ten-inch photomultiplier tubes with a titanium cylinder housing the electronicd. The distance between detection lines is 70 metres. Vertically a line is a set of 25 storeys separated by 14.5 metres, resulting in a total height of 450 metres. The instrumented detector volume is about of $0.02 \mathrm{~km}^{3}$. A sketch of the ANTARES telescope is displayed on Figure Antares.

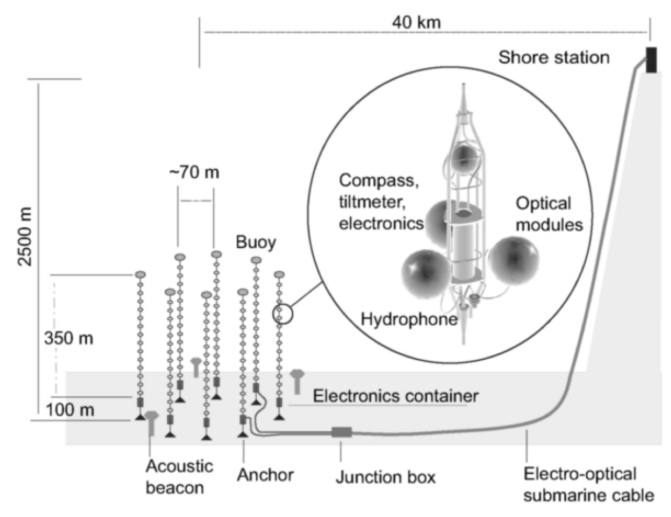

Figure 1: Sketch of the ANTARES telescope, consisting of a tridimensional array of 900 PMTs distributed along 12 lines, anchored at the bottom of the Mediterranean Sea at $2400 \mathrm{~m}$ deep.

Before its complete installation released in May 2008, ANTARES has been taking data in intermediate configurations of 5 and 10 detector lines for more than one year. The angular resolution 
of the telescope is of the order of one degree at low neutrino energy (typically $E_{v}<1 \mathrm{TeV}$ ) which is relevant to dark matter searches.

\section{Neutralino annihilation in the Sun}

In order to perform the estimation of the $v_{\mu}+\bar{v}_{\mu}$ flux resulting from neutralino annihilation in the centre of the Sun, a more constrained approach of minimal supergravity (mSugra) has been followed, instead of the general supersymmetry scenario. Such mSugra models are driven by only four parameters $m_{1 / 2}, m_{0}, A_{0}, \tan (\beta)$ and also a sign of $\mu$ : $\operatorname{sgn}(\mu)$. A local neutralino halo density of $0.3 \mathrm{GeV} / \mathrm{cm}^{3}$ has been assumed and the DarkSUSY package [2] combined with the renormalisation group evolution package ISASUGRA [3] has been used. A dedicated attention has been done to investigate those mSUGRA models where a relic neutralino density $\Omega_{\chi}$ is compatible with the cold dark matter density $\Omega_{\chi}$,WMAP as measured by WMAP [4]. Then using a random walk method in mSUGRA parameter space based on the Metropolis algorithm [5], the neutrino flux has been calculated for four million mSUGRA models. Only positive sign of $\mu$ models have been considered while the parameters stand in the following ranges: $0<m_{0}<8000 \mathrm{GeV}, 0<m_{1 / 2}<2000 \mathrm{GeV}$, $-3 m_{0}<A_{0}<3 m_{0}$ and $0<\tan (\beta)<60$. The $v_{\mu}+\bar{v}_{\mu}$ flux from the Sun, integrated above a energy of $E_{v}=10 \mathrm{GeV}$ is displayed in Fig. 2 where the $m_{0}-m_{1 / 2}$ plane is given for six different $\tan (\beta)$ ranges. The highest solar neutrino flux are produced in the so-called focus point region, where the neutralino has a relatively large higgsino component and then a large neutralino vector-boson coupling.
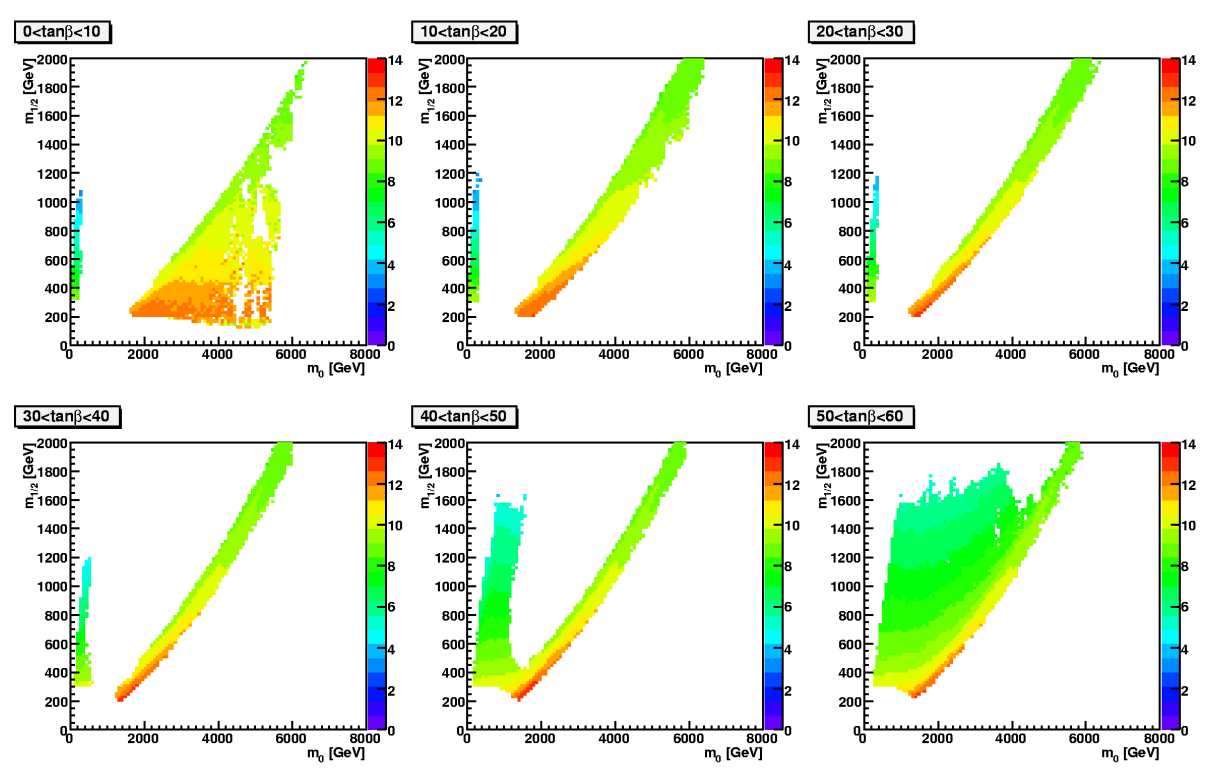

Figure 2: The $\log _{10}\left(v_{\mu}+\bar{v}_{\mu}\right)$ flux from neutralino annihilation in the Sun $\left[\mathrm{km}^{-2} \mathrm{year}^{-1}\right]$ in $m_{0}-m_{1 / 2}$ mSUGRA parameter space, for six different $\tan (\beta)$ intervals. $A_{0}$ varies between $-3 m_{0}$ and $3 m_{0}$. The flux was integrated above $E_{v}=10 \mathrm{GeV}$. 


\section{Expected detection sensitivity}

The ANTARES detection sensitivity for neutralino annihilation in the Sun was determined by considering the irreducible background from atmospheric neutrinos and an additional $10 \%$ of that flux due to misreconstructed atmospheric muons in a search cone of 3 degree radius around the Sun [6]. Based on the ANTARES effective area displayed on Fig. 3, the average background prediction after 3 years of effective data taking is $\sim 7$ neutrinos.

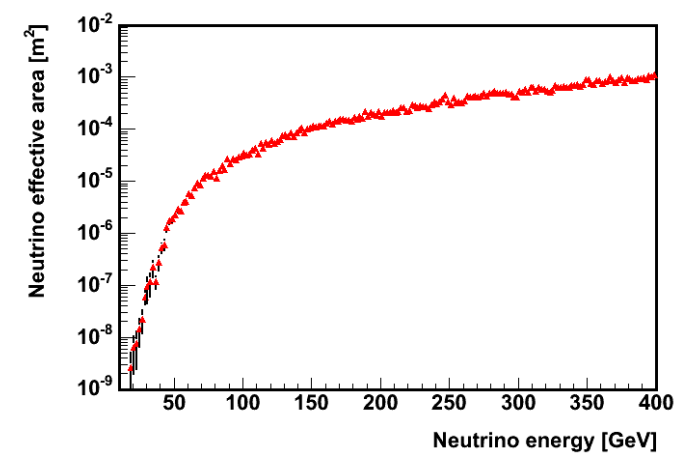

Figure 3: The 12 line ANTARES effective area.

According to the "G. J. Fledman and R. D. Cousins" procedure [8], a 90\% CL upper limit on the neutrino flux from the Sun for 3 years of effective data taking may be derived. The ANTARES detection sensitivity is displayed in Fig. 4 in the $m_{0}-m_{1 / 2}$ mSUGRA paramater plane for different $\tan (\beta)$ intervals.
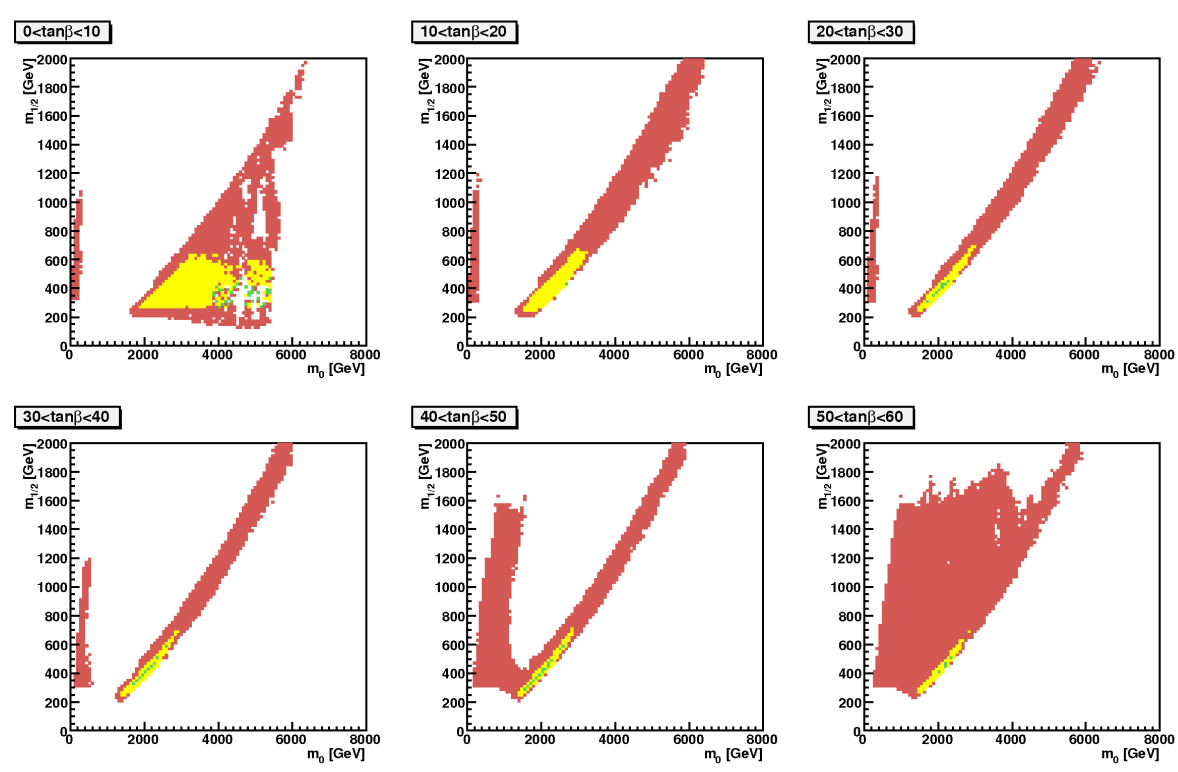

Figure 4: Sensitivity of ANTARES (12 lines) in $m_{0}-m_{1 / 2}$ mSUGRA parameter space, for six different $\tan (\beta)$ intervals. $A_{0}$ varies between $-3 m_{0}$ and $3 m_{0}$. Green/yellow/red indicates that all/some/no mSUGRA models (depending on $A_{0}$ and $\tan (\beta)$ ) can be excluded at $90 \%$ CL after 3 years. 
The different colours (Green/yellow/red) indicate respectively that all/some/no mSUGRA models (depending on $A_{0}$ and $\tan (\beta)$ ) can be excluded at $90 \% \mathrm{CL}$ after 3 years of effective data taking. The sensitivity of ANTARES is sufficient to put constraints on parts of the focus point region of mSUGRA parameter space.

The KM3NeT consortium aims to build a cubic-kilometer scale neutrino telescope in the Mediterranean Sea [7]. The reference detector consists of a cubic configuration made of of 225 detection lines resulting in an instrumented detector volume of $1 \mathrm{~km}^{3}$. Following a similar computation, the results for the KM3NeT detector are displayed in Fig. 5. KM3NeT would be sensitive to most of the focus point region of mSUGRA parameter.
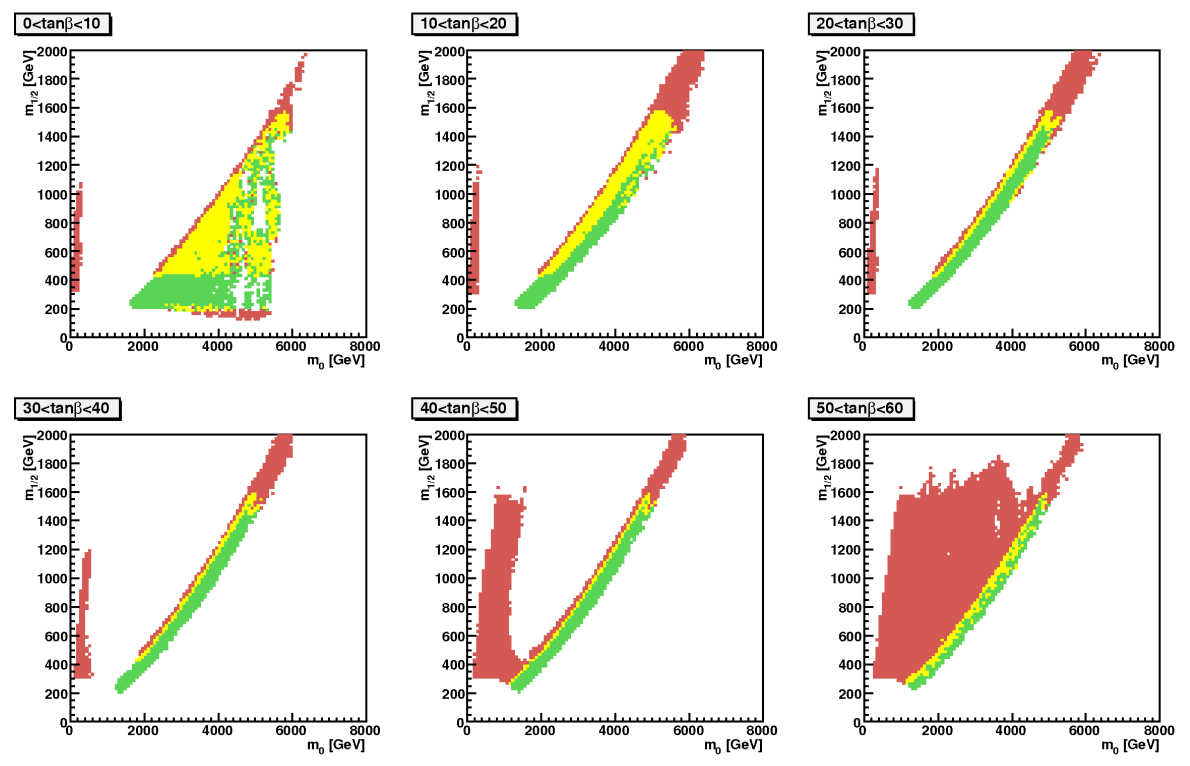

Figure 5: Sensitivity of KM3NeT in $m_{0}-m_{1 / 2}$ mSUGRA parameter space, for six different $\tan (\beta)$ intervals. $A_{0}$ varies between $-3 m_{0}$ and $3 m_{0}$. Green/yellow/red indicates that all/some/no mSUGRA models (depending on $A_{0}$ and $\tan (\beta)$ ) can be excluded at $90 \% \mathrm{CL}$ after 3 years.

\section{Data analysis}

Search for a possible excess in the neutrino flux from the Sun has been performed in the data taken in 2007 during the operation of the first 5 lines setup corresponding to an effective livetime of 68.4 days taking into account the detector dead-time and the condition that the Sun has to be below the horizon. The number of observed neutrinos in a search cone around the Sun direction is displayed in Fig. 6(left) as a function of the search cone radius. The expected number of background events estimated by Monte Carlo simulation is in good agreement with the estimation obtained by randomising the direction and arrival time of the observed events. The upper limit and sensitivity on the number of signal events according to "G. J. Fledman and R. D. Cousins" procedure [8] is also reported. The corresponding upper limit on the neutrino flux from the Sun may be derived as a function of the neutralino mass. Two extreme annihilation cases have been considered: Pure annihilation into vector-bosons and into $b \bar{b}$ quarks only, so-called respectively "hard" and "soft" annihilation. In both cases, the neutrino energy spectra at Earth as a function of the neutralino mass 
was estimated with the WimpSim [9] package, assuming the standard oscillation scenario. The resulting upper limit at $90 \% \mathrm{CL}$ on the neutrino flux from the Sun, integrated above an energy of $E_{v}=10 \mathrm{GeV}$ is displayed in Fig. 6(right). The expected neutrino flux from the mSUGRA models considered is also displayed according to the compatibility of their $\Omega_{\chi}$ to $\Omega_{\chi}$,WMAP.
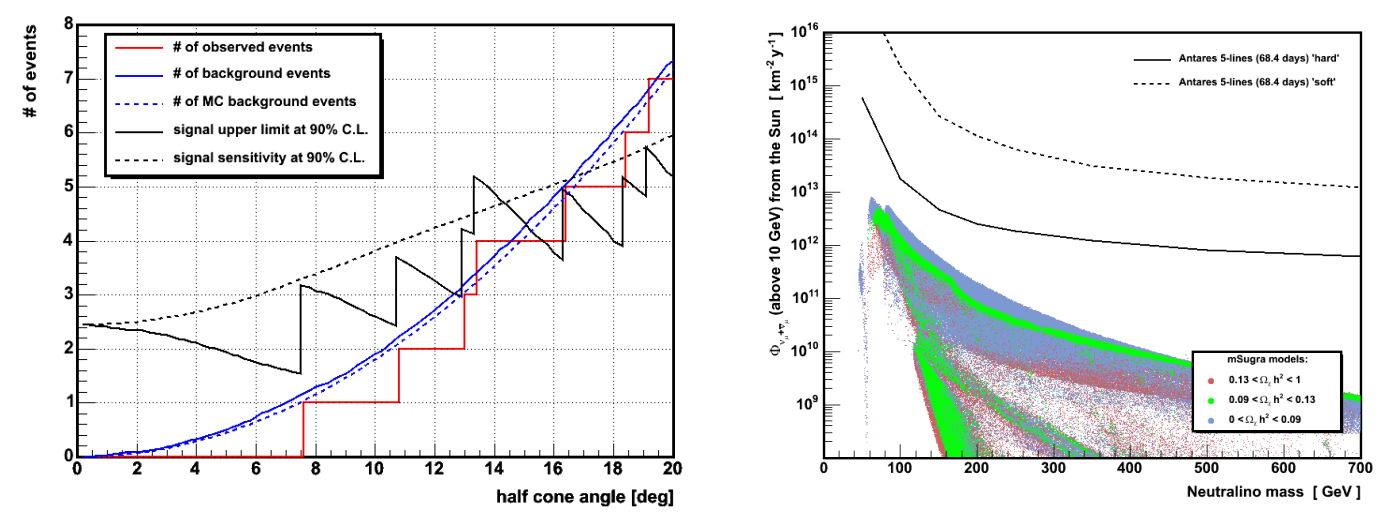

Figure 6: (left) Number of observed neutrinos and expected background events as a function of the search cone radius around the Sun. (right) Upper limit on the neutrino flux from the Sun above $E_{v}=10 \mathrm{GeV}$ and the corresponding muon flux above $E_{\mu}=1 \mathrm{GeV}$ for the 5-line ANTARES period as a function of the neutralino mass, in comparison to the expected flux from the mSUGRA models. Models indicated in green lie within $2 \sigma$ of the preferred WMAP value, while models in red/blue have a higher/lower relic density.

\section{Conclusion}

The ANTARES detector is fully operational since July 2008. First upper limits of the ANTARES detector in its intermediate 5 line configuration on the neutrino and muon flux from neutralino annihilation in the Sun have been derived. Monte Carlo simulations based on the full ANTARES detector and a possible configuration of the future KM3NeT detector show that those apparatus will be able to probe the Focus Point region of the mSUGRA parameter space.

\section{References}

[1] J. Aguilar et al. , Astropart. Phys. 26, 314 (2006); J. Carr, J. Phys.: Conf. Ser. 136, 022047 (2008); M. Ageron et al. , Astropart. Phys. 31, 277 (2009)

[2] P. Gondolo et al. , JCAP 0407, 8 (2004)

[3] H. Baer et al. , arXiv: hep-ph/0312045

[4] WMAP collaboration, Astrophys. J. 148, 175 (2003)

[5] E. Baltz, P. Gondolo, JHEP 10 (2004) 52

[6] G. Lim et al. , ICRC Proceedings, Łódź (2009)

[7] KM3NeT CDR (2008), http: / / www. km3net. org; U. Katz, Nucl. Instr. Meth. A 602, 40 (2009)

[8] G. Feldman, R. Cousins, Phys. Rev. D 57, 3873 (1998)

[9] M. Blennow et al. , JCAP 0108, 21 (2008) 